\title{
Entre cuotas y violencia de género: avances y retrocesos en la participación política de las mujeres en México*
}

\author{
Karolina M. Gilas \\ Alma Verónica Méndez Pacheco**
}

Recibido: 15 de agosto de 2017

Evaluado: 31 de enero 2018

Aceptado: 16 de abril de 2018

\section{Resumen}

Las cuotas de género son una medida positiva que tienen como propósito incrementar la representación política de las mujeres. En el caso mexicano, su implementación comenzó en la década de los noventa y su evolución ha producido, en los últimos años, además de un $42.6 \%$ de la representación femenina en el Congreso federal, un incremento considerable en la violencia política de género. El presente artículo tiene como objetivo exponer, por una parte, el impacto positivo de la judicialización de la política en México en la representación política de las mujeres y, al mismo tiempo, explicar la relación entre estas medidas y la violencia política contra las mujeres.

Palabras clave: cuotas de género, violencia política de género, México, elecciones.

** Doctora en Ciencia Política por la Universidad Nacional Autónoma de México. Asesora en el Tribunal Electoral del Poder Judicial de la Federación. Correo electrónico: karolina_gilas@hotmail.com

*** Licenciatura en Ciencias de la Comunicación por la Universidad Nacional Autónoma de México. Asistente de investigación en el Tribunal Electoral del Poder Judicial de la Federación. Correo electrónico: veroumz@gmail.com 


\title{
Between gender quotas and political violence: progress and setbacks in political representation of women in Mexico
}

\begin{abstract}
Gender quotas are a specific type of positive actions aimed at fostering women's political representation. In Mexico, gender quotas were adopted since 1993 and over the years their evolution produced a federal legislature with $42.6 \%$ of women, but also a rise of political violence against women. This paper explains the highly positive impact of judicialization on women's representation and analyses the relation that seems to appear between effectively applied gender quotas and an increase in political violence against women.
\end{abstract} Received: August 15, 2017 Evaluated: January 31, 2018 Accepted: April 16, 2018

Keywords: gender quotas, political violence against women, Mexico, elections. 


\title{
Entre cotas e violência de gênero: avanços e retrocessos na participação política das mulheres no México
}

\begin{abstract}
Resumo
As cotas de gênero são uma medida positiva que tem o objetivo de aumentar a representação política das mulheres. No caso mexicano, a sua implementação começou na década de noventa e sua evolução tem ocorrido nos últimos anos, mais de $42.6 \%$ de representação feminina no Congresso federal, um aumento considerável na violência política de gênero. $\mathrm{O}$ objetivo deste artigo é expor, por um lado, o impacto positivo da judicialização da política no México sobre a representação política das mulheres e, ao mesmo tempo, explicar a relação entre essas medidas e a violência política contra as mulheres.

Palavras-chave: cotas de gênero, violência de gênero, México, eleições.
\end{abstract}




\section{INTRODUCCIÓN}

A lo largo del desarrollo de las civilizaciones occidentales, los patrones culturales, sociales y legales han mantenido una clara división sexuada de la población, en la que las mujeres fueron identificadas con la naturaleza y las emociones y restringidas al ámbito privado, mientras que a los hombres se les reconocía la capacidad de pensamiento racional y de abstracción, destinándolos al ámbito público y a la toma de decisiones. El reconocimiento del sufragio femenino otorgó a las mujeres la capacidad de participar en la toma de decisiones colectivas, pero no fue de la mano de la modificación de los estereotipos y los roles de género. En consecuencia, la capacidad de las mujeres de ejercer su derecho al sufragio pasivo y participar en los espacios de representación y de toma de decisiones siguió siendo limitada.

Ese hecho motivó a la mayoría de los países democráticos a buscar mecanismos que permitieran cerrar la brecha de género y fortalecer la representación femenina. Así, en la década de los noventa empezó la implementación de las cuotas de género, que se extendió rápidamente, especialmente en el continente americano ${ }^{1}$. México fue uno de los países que entró a la ruta de las cuotas.

El caso mexicano es particularmente interesante, en por lo menos dos aspectos. El primero, es que la evolución de las cuotas en México confirma los hallazgos de la literatura especializada que señalan que la efectividad de la cuota depende de su diseño, que

Argentina fue el primer país de América Latina en implementar la cuota de género, en 1991. Siguieron México, Costa Rica, Paraguay, Bolivia, Brasil Ecuador, Panamá, República Dominicana, Brasil, Perú, Venezuela y Honduras (Krook, 2008, p. 16). debe ser compatible con el sistema electoral, y que debe contener medidas efectivas en su implementación, usualmente sanciones por el incumplimiento (Dahlerup y Freidenvall, 2005; Norris, 2006). El segundo, es que la evolución de las cuotas en México, si bien ilustra la dinámica descrita por la literatura, tiene un rasgo particular: el papel de los órganos jurisdiccionales en el desarrollo de las cuotas (González, Gilas y Báez, 2016).

El presente artículo tiene como objetivo explicar el impacto positivo de la judicialización de la política en México en la representación política de las mujeres, centrándose en el desarrollo jurisprudencial posterior a la reforma electoral de 2014, que reconoció la paridad de género como un principio constitucional. Asimismo, pretende esbozar una problemática que ha estado presente en los últimos años, como reacción negativa al incremento de la presencia femenina en la política: la violencia política de género.

\section{Cuotas de género}

La exclusión de las mujeres de una ciudadanía efectiva fue un fenómeno generalizado hasta, por lo menos, finales del siglo XIX. En la expectativa de las feministas, es con el reconocimiento del voto femenino que se iba a lograr la ciudadanía plena para las mujeres (Schnapper, 2004; Lau Jaiven, 2013). Aunque la realidad política y social fue otra, ya que el reconocimiento del sufragio no conllevó a una mayor representación política ni participación en los espacios de decisión de las mujeres. Por ello, especialmente desde la década de 1990, el tema de la igualdad en la participación política entre mujeres y hombres cobró especial relevancia a nivel internacional, muestra de ello 
fueron los programas como la Declaración de Atenas de 1992 o la Declaración de Beijing y su Plataforma para la acción de 1995, signados por diversos países, donde se establecían acciones específicas para alcanzar una igualdad sustancial entre los géneros.

Entre los primeros mecanismos que se implementaron para reducir la brecha de desigualdad, se encuentran las cuotas de género, una medida positiva que ha tenido un impacto distinto de acuerdo con el país donde se ha adoptado, su sistema electoral, así como su nivel de compromiso (IDEA, 2002, p. 17). En algunos países las cuotas empezaron a utilizarse desde los años setenta, aunque en el continente americano y en México su incorporación llegó apenas en la década de los noventa (Medina Torres, 2011, p. 9). En ese contexto, la introducción de las cuotas fue resultado del lento incremento "natural" de la representación política de las mujeres y de las presiones ejercidas por las organizaciones feministas e internacionales (Dahlerup y Freidenvall, 2005, pp. 29-30).

Las cuotas de género,

[...] buscan elevar el porcentaje de mujeres en el Parlamento o alcanzar el equilibrio de género y establecen una participación mínima de candidatas en las elecciones, por lo menos en las listas de los partidos. Adicionalmente, pueden también contener disposiciones que toquen el posicionamiento de candidatas en las listas (Krennerich 2009, p. 189).

En función de los objetivos a lograr y de las particularidades de cada sistema electoral, el diseño de las cuotas llega a ser muy diverso. Sin embargo, existen dos variables principales en la tipología de las mismas: su nivel de introducción y su nivel de aplicación. En ese sentido, podemos distinguir entre las cuotas legisladas (a nivel constitucional o en la legislación ordinaria) y aprobadas voluntariamente por los partidos, así como entre las cuotas que pretenden regular la postulación de las mujeres entre los precandidatos, entre los candidatos o entre las personas electas (Dahlerup, 2006, p. 19).

Las cuotas de género resultaron ser el mecanismo más efectivo y rápido para lograr un incremento cuantitativo significativo en la representación política de las mujeres. Por ejemplo, en Costa Rica se logró elevar la representación femenina del 19 al $35 \%$ en una sola elección. Por supuesto, el éxito de las cuotas depende de su diseño. Estas deben ser adecuadas en función del sistema electoral existente, ya que los sistemas proporcionales, mayoritarios o mixtos tienen características particulares que requieren ajustar las medidas positivas. Además, de acuerdo con Dahlerup y Freidenvall (2005 y 2008), existen otros dos factores clave para la efectividad de las cuotas: su obligatoriedad y la existencia de sanciones efectivas por su incumplimiento. En el siguiente apartado evaluaremos, con la evolución de la cuota en México, qué tan acertada es esa afirmación.

\section{De las cuotas de género a la PARIDAD Y LA JUDICIALIZACIÓN DE LA POLítica en MÉxico}

La transición mexicana a la democracia fue un proceso centrado en el diseño e implementación de la competitividad electoral. Esa dinámica derivó del diagnóstico particular del sistema político del país, bajo el 
cual el diseño institucional correspondía a un Estado democrático, por lo que los únicos elementos "faltantes" eran un sistema fuerte de partidos y una real competencia electoral. En función de ello, el país pasó por una serie de reformas, cuyo objetivo fue la creación de autoridades electorales independientes, capaces de organizar procesos electorales limpios y competidos, así como la institucionalización de un sistema plural de partidos (Santiago Castillo, 2011; Woldenberg, 2012).

Parte importante de esa evolución fue la creación de un órgano especializado encargado de la resolución de los conflictos electorales. Los setenta años de un gobierno autoritario que organizaba las elecciones cuyos ganadores eran conocidos antes de la jornada electoral, generaron en la oposición y en la sociedad mexicana una fuerte desconfianza hacia las instituciones responsables de la organización de los procesos electorales, su calificación y resultados. Esa desconfianza está en el origen del sistema electoral mexicano, altamente regulado, y en el comienzo de la búsqueda de un árbitro independiente. Los elementos centrales de ese diseño fueron creados con la reforma electoral de 1990, con la creación del Instituto Federal Electoral (IFE) y su contraparte, el Tribunal Federal Electoral (TRIFE). El primero, encargado de la organización de las elecciones, en tanto el segundo, de la resolución de los conflictos electorales. Más tarde, en 1996, el IFE pasó por un proceso de ciudadanización, mientras que el TRIFE se convirtió en el Tribunal Electoral del Poder Judicial de la Federación, (TEPJF), el órgano jurisdiccional máximo en materia electoral
(Woldenberg, 2012) ${ }^{2}$. La cantidad y complejidad de los procesos electorales en México y frecuentes reformas a la legislación electoral, convirtieron al TEPJF en un actor clave, cuya función fue más allá de la resolución de conflictos electorales, abarcando también la constante interpretación de las leyes y el control de constitucionalidad de los actos de las autoridades ${ }^{3}$. Así, uno de los ámbitos en los que la actuación del Tribunal ha tenido mayor impacto es en la implementación de las cuotas de género.

En el caso de México, las cuotas de género se introdujeron en la legislación electoral de 1993, aunque con carácter de recomendación a los partidos políticos: "Los partidos políticos promoverán, en los términos que determinen sus documentos internos, una mayor participación de las mujeres en la vida política del país, a través de su postulación a cargos de elección popular" (COFIPE, artículo 175.3, 1993). Era de suponerse que una cuota de género tan laxa no implicaría mayor cambio en el sistema político mexicano, por lo que la reforma político-electoral de 1996 introdujo una disposición un tanto más fuerte, al establecer que ningún partido político podría postular más del $70 \%$ de candidatos de un solo género, aunque la ley no contemplaba una sanción en caso del incumplimiento de la norma. Más tarde, la reforma del 2002 mantuvo la relación 70-30 $\%$ en la postulación de candidatos, no obstante, estableció que el cumplimiento solo

2 Dicha estructura, de una autoridad administrativa y una jurisdiccional, se replicó a nivel local en cada una de las entidades federativas.

3 El número de casos por año que el TEPJF ha resuelto desde su inicio, se ha incrementado exponencialmente: de 19 casos en 1996 a 35.628 en 2011, su punto más álgido; hasta noviembre de 2017 había resuelto 8.228 asuntos (TEPJF, 2017). Lo que muestra que los asuntos en los que el Tribunal tiene que pronunciarse se incrementan de forma constante. 
debía aplicarse a los propietarios (principales) de las fórmulas y no a los suplentes, lo cual derivó en diversas tácticas por parte de los partidos políticos para evadir las cuotas de género (cabe señalar que esa regla deriva de la interpretación del Tribunal). Es en esa etapa cuando el TEPJF empieza a pronunciarse sobre la implementación de las cuotas, decidiendo, por ejemplo, la obligatoriedad de la cuota para los partidos políticos y la legalidad de las modificaciones o sustituciones de candidatos en las listas ya registradas, de ser necesarias para el cumplimiento de la cuota (González et al., 2016).

Más tarde, la reforma de 2008 incrementó del 30 al $40 \%$ la presencia obligatoria de mujeres en las candidaturas y, aunque mantuvo la excepción de la cuotas a los candidatos por el principio de mayoría relativa si estos eran elegidos mediante un proceso democrático al interior de sus partidos (COFIPE, artículo 219.2, 2008), modificó la manera de integrar las listas por el principio de representación proporcional: en segmentos de 5 candidatos alternando los géneros, por lo que cada segmento no podía tener más de 3 candidatos de un mismo género.

En la práctica política, la excepción prevista por la legislación limitaba la aplicación de la cuota únicamente al ámbito de las postulaciones por la vía de representación proporcional (el 40 \% de los escaños en la Cámara de Diputados y una proporción similar en las legislaturas estatales), ya que los candidatos por la vía de mayoría relativa solían ser nombrados a través de un "proceso democrático". Aun así, en esa etapa de aplicación de las cuotas, la interpretación del Tribunal Electoral resultó ser clave para su implementación, por ejemplo, para el respeto al principio de alternancia en las listas de candidatos y del sufragio efectivo de las mujeres en las elecciones por usos y costumbres ${ }^{4}$.

No fue sino hasta 2011, con la Sentencia SUP-JDC-12624/2011, conocida como "anti-juanitas", que el TEPJF eliminó la excepción a las cuotas de género y estableció la obligatoriedad de que el $40 \%$ de las fórmulas reservadas a las mujeres estuvieran compuestas por propietaria (principal) y suplente del mismo género. Si bien:

[...] la primera consecuencia, y la más visible, de la Sentencia SUPJDC-12624/2011 fue el incremento de la presencia femenina en ambas cámaras del Congreso. En la elección de 2012 se alcanzaron los niveles históricos de $37.4 \%$ en la Cámara de Diputados y de 33.6 \% en el Senado (González, et al., 2016, p. 139).

Lo cierto es que fue el parteaguas en la discusión sobre la igualdad sustancial en la participación política de las mujeres frente a los hombres.

Así, la reforma de 2014 introdujo la paridad de género a nivel constitucional y derivó en diversos cambios a nivel legal:

Los partidos políticos tienen como fin promover la participación del pueblo en la vida democrática, contribuir a la integración de los órganos de representación política y como organizaciones

4 Por mencionar algunos casos: la Sentencia SUP-JDC-2580/2007 y sus acumulados SUP-JDC-1/2008 y SUP-JDC-12/2008 donde el Tribunal estableció que las listas a diputaciones por representación proporcional debían integrarse por 12 fórmulas, con máximo el $70 \%$ de candidatos propietarios de un mismo género, en segmentos de tres candidaturas con un género distinto. 


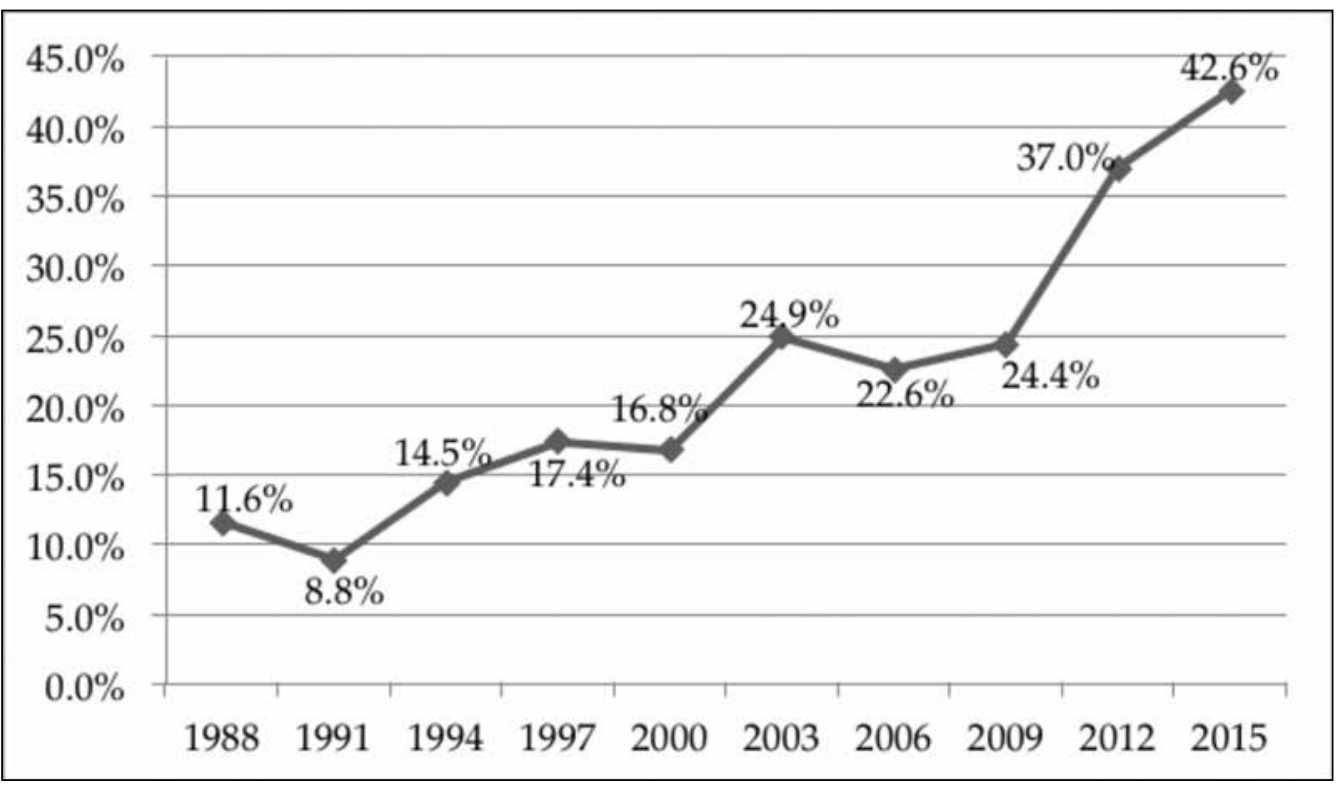

Figura 1. Presencia de las mujeres en la Cámara de Diputados

Fuente: elaborado con base en González, et al., 2016.

de ciudadanos, hacer posible el acceso de estos al ejercicio del poder público, de acuerdo con los programas, principios e ideas que postulan y mediante el sufragio universal, libre, secreto $y$ directo, así como las reglas para garantizar la paridad entre los géneros, en candidaturas a legisladores federales y locales (CPEUM, artículo 41, Base I).

A continuación se enlistan los principales cambios introducidos por la reforma legal en la materia:

- Los partidos políticos tienen la obligación de postular a sus candidatos a los poderes legislativos federal y locales en una proporción de $50 \%$ mujeres y $50 \%$ hombres, por ambos principios (LEGIPE, artículo 232.3).

- Cada partido determinará y hará públicos los criterios para garantizar que la paridad de género se cumpla (LGPP, artículo 3.3 y 3.4).
- Se prohíbe que los distritos perdedores, es decir, donde los partidos políticos hayan obtenido menor votación en la elección anterior inmediata se asignen exclusivamente a un género (LGPP, artículo 3.5).

- La paridad de género debe observarse incluso en las elecciones bajo sistemas normativos internos (LEGIPE, artículo 26.4).

- Las fórmulas de candidatos deben ser del mismo género y en el caso de los candidatos a senadores se debe registrar una lista con dos fórmulas, cada una de un mismo género (LEGIPE, artículo 14.3 y 14.4).

- En la integración de las listas de representación proporcional deben alternarse las fórmulas, integradas por propietario (principal) y suplente del mismo género, hasta agotarse cada lista (LEGIPE, artículo 234).

- Tanto el INE, como los Oples están facultados para rechazar el registro de las listas de candidatos de 
los partidos que no cumplan con lo establecido en la ley, aunque deben otorgar un plazo para que se subsanen tales faltas y de no hacerlo, negar definitivamente el registro (LEGIPE, 232.4).

- Los partidos políticos deben destinar el $3 \%$ de su presupuesto anual para fomentar y fortalecer el liderazgo de las mujeres (LGPP, 51.1, inciso a), base V).

El proceso electoral 2014-2015 -por el cual se renovó la Cámara de Diputados federal y se llevaron a cabo procesos concurrentes en 17 entidades federativas-, fue la prueba a la reforma electoral. Durante este se presentaron cientos de casos derivados de la aplicación e interpretación de la paridad de género. Las interpretaciones del Tribunal emitidas en 2015, así como la consolidacion de los criterios durante las elecciones de 2016, resultaron claves para la implementación de nuevas reglas de paridad en todas las elecciones populares.

\section{Cumplimiento de la paridad en el registro de candidaturas}

Uno de los casos más interesantes sobre el cumplimiento de la paridad de género en el registro de candidaturas se presentó en 2015 en Chiapas, donde el Instituto Electoral local registró las postulaciones de los partidos políticos incluso cuando estos no cumplieron con la paridad.

Inicialmente, la Sala Regional Xalapa del TEPJF tuvo conocimiento del caso, pero sin embargo, argumentó que debido a lo avanzado del proceso electoral no se podían modificar las listas de los partidos, aunque sí amonestó a quienes incumplieron con la norma constitucional. Dicha resolución fue impugnada ante la Sala Superior por el partido Movimiento Ciudadano, quien adujo que el inicio de las campañas electorales no se traducía en un obstáculo para que se resolviera el fondo de la controversia (SUP-REC-294/2015).

Al respecto, la Sala Superior sostuvo que era "inadmisible el incumplimiento abierto a las normas constitucionales y legales [...] toda vez que su contenido normativo era conocido y, por tanto, estaba llamado a regir la conducta de los partidos políticos y a las autoridades electorales locales" (SUPREC-294/2015). De modo que dictó un plazo de 48 horas para que los partidos políticos que se encontraran en ese supuesto, modificaran sus listas. Ante la premura -ya que la Sala Superior resolvió el asunto el 8 de julio y la jornada electoral se celebraba el 19 de julio-, algunos partidos ya no modificaron sus listas, por lo que no pudieron contender en al menos un municipio; este fue el caso de los partidos Humanista, Encuentro Social, Verde Ecologista de México y Nueva Alianza (González, 2015).

\section{Postulación de mujeres en distritos con menor votación o "perdedores"}

Como se mencionó con anterioridad, la reforma electoral estableció entre sus reglas la prohibición de que a alguno de los géneros se le asignen exclusivamente las candidaturas en los distritos donde el partido político haya obtenido la menor cantidad de votos en el proceso electoral anterior (LGPP, artículo 3.5).

De la revisión de las leyes locales se puede afirmar que de los 18 procesos electorales durante 2014-2015, solo en la mitad se reguló al respecto de esta norma. E incluso, el hecho de introducir explícitamente la 
observancia de la norma en sus leyes locales no significó que en todos los Estados se revisara su cumplimiento. De acuerdo con la revisión de los acuerdos emitidos por los organismos públicos locales, solo un tercio de dichas entidades federativas comprobó el cumplimiento del artículo 3.5 de la Ley General de Partidos Políticos para integrar los poderes legislativos.

A nivel federal, el INE emitió el Acuerdo INE/CG162/2015 mediante el cual el Consejo General del INE registró las candidaturas a diputados federales y definió la metodología para analizar el cumplimiento de lo establecido por el artículo 3.5 de la LGPP. Así, clasificó los distritos en tres: distritos ganadores o de mayor votación, distritos intermedios o de votación media y distritos perdedores o de menor votación. Esta metodología fue avalada por la Sala Superior del TEPJF al resolver la Sentencia SUP-RAP-134/2015 y, a partir de esta clasificación, el INE comprobó que los partidos políticos no registraran una mayor cantidad de candidatas en los distritos perdedores, bajo el criterio de que el concepto de exclusividad no debía entenderse como totalidad, porque ello "posibilitaría la inaplicación de la disposición aludida mediante la designación de un solo candidato del género contrario a dichos principios, lo que violentaría el principio que dicha norma tutela" (INE/CG162/2015). Así, el INE interpretó que en la postulación no debía haber un sesgo evidente hacia alguno de los géneros. Este fue también el caso de Colima, donde la Sala Toluca aplicó la metodología del INE para comprobar que ninguno de los géneros fuera afectado exclusivamente con la postulación en distritos perdedores (ST-JRC-21/2015).
En contraste, en los Estados de Chiapas, Nuevo León y Morelos, los distritos se dividieron solo en 2: ganadores y perdedores. En el caso de Chiapas y Nuevo León, se verificó tanto en los distritos perdedores como en los ganadores que se cumpliera con la postulación paritaria en proporción de 50-50 (Anexo 1 del Acuerdo IEPCO/CG/A-081-2015 y lineamientos y formatos generales para el registro de las candidatas y los candidatos del año 2015). En tanto en Morelos, solo se revisó que en los distritos perdedores se postulara el mismo número de candidatos de ambos géneros (IMPEPAC/CEE/035/2015).

Si bien en el Estado de México el organismo público local no comprobó el cumplimiento de la norma de forma general, se presentó un caso donde un militante impugnó la lista de candidatos a diputados locales del Partido de la Revolución Democrática (PRD). Tras el análisis de los distritos ganadores, intermedios y perdedores, la Sala Toluca determinó que, si bien formalmente el partido había cumplido con la distribución paritaria de las candidaturas por mayoría relativa, incurrió

[...] en una disparidad en la asignación de las candidaturas, merced a una correlación inversamente proporcional: hay más candidaturas a mujeres en distritos en donde, en función de la escaza votación precedente, puede resultarles más difícil tener una participación efectiva y consecuentemente acceder al cargo público, pues el partido no ha sido competitivo $y$, en la misma medida les resulta más difícil a las mujeres concretizar su plena participación porque también se les limita la asignación de candidaturas 
en distritos más accesibles a un eventual triunfo en virtud a lo copioso de los votos al partido político postulante (ST-JDC-278/2015, ST-JDC-279/2015 y ST-JDC-280/2015).

Lo anterior, porque de los 8 distritos con menor cantidad de votos, en 5 habían sido postuladas mujeres, por lo que revocó la decisión tomada por el partido y lo instó a cumplir con la norma.

\section{Conformación de listas de representación proporcional e integración de los órganos electos}

Durante el proceso electoral 2014-2015, otro tema de discusión fue la distribución de los escaños de los órganos electos al respecto de llevar la paridad al nivel de modificar las listas registradas por los partidos políticos de los candidatos por el principio de representación proporcional (RP), para compensar con ello la disparidad en los resultados obtenidos en los distritos uninominales.

Si bien el Estado de Coahuila no tuvo elecciones en 2015, la integración de su Congreso en 2014 fue el primero que planteó esta disyuntiva al TEPJF, ya que pese a alcanzar una integración paritaria con los triunfos por mayoría relativa, al asignar los escaños de RP el Congreso quedó con 14 hombres y 11 mujeres, por lo que la Sala Monterrey modificó el orden de las listas de los partidos para que la integración final fuera de 13 mujeres y 12 hombres. Lo anterior bajo el argumento de que:

No se priva de un derecho adquirido al candidato que ocupe el primer lugar de la lista, pues con independencia de la posición que tenga en el listado, su derecho a detentar un escaño estará limitado en la medida que su nombramiento como diputado impida que, en la integración del Congreso del Estado, se respete el principio de igualdad y así como las acciones afirmativas en materia de equidad de género (SM-JRC-14/2014).

Si bien este criterio fue confirmado por la Sala Superior en el recurso de reconsideración SUP-REC-936/2014, más tarde la Máxima Sala del TEPJF consideró que el principio de paridad se cumplía con la postulación de los candidatos, y al integrar las listas de RP de forma alternada y que

la conformación paritaria de los órganos deliberativos de elección popular, se define por el voto ciudadano, ya que son los electores quienes eligen a las candidaturas de sus preferencias de entre aquellas que participan en la contienda electoral en un porcentaje igualitario de cada género -cincuenta por ciento de mujeres y cincuenta por ciento de hombres- (SUP-JRC-680/2015).

Además, la Sala Superior sostuvo que corresponde al poder reformador de la Constitución establecer que la paridad de género debe aplicarse también en la integración de los órganos de representación popular (SUP-JDC-1236/2015). Este criterio fue confirmado en diversos asuntos, entre los que se encuentran la asignación de diputaciones locales por el principio de representación proporcional en el Estado de Colima y en San Luis Potosí (SUP-REC-756/2015 y SUP-REC-762/2015, acumulados; SUPREC-694/2015). 


\section{El impacto de la paridad}

Tras la aplicación de las nuevas normas electorales en materia de paridad, en los procesos electorales de 2014-2016, las mujeres generalmente obtuvieron una mayor representación en los Congresos locales y en la Cámara de Diputados federal.

En un ejercicio comparativo entre los resultados electorales de 2012 y 2015, se muestra un incremento considerable en el número de mujeres que integran el poder legislativo mexicano, del 24.6 al $41.5 \%$ en promedio, es decir, un incremento del $16.8 \%$, a excepción del Estado de Morelos, donde hubo una disminución del $3.3 \%$ (véase Figura 2).

En cuanto a los resultados electorales del 2013 frente a los del 2016, el incremento en la representación femenina fue del 28.9 al 38.5
$\%$ en promedio, es decir, un incremento de $9.6 \%$, siendo Hidalgo, Tamaulipas, Veracruz y Zacatecas los Estados donde la presencia de las mujeres disminuyó en comparación con el proceso electoral anterior (véase Figura 3).

Asimismo, durante el proceso electoral de 2014-2015, se observó una diferencia sustancial a nivel municipal, en comparación con procesos anteriores. De los 871 municipios que renovaron sus autoridades, fueron electas 105 mujeres, 66 de las cuales fueron elegidas como presidentas municipales. Si bien el número sigue siendo mínimo en comparación con los hombres, representó un avance del $14.8 \%$ en comparación con 2012 (Observatorio de la Participación Política de las Mujeres).

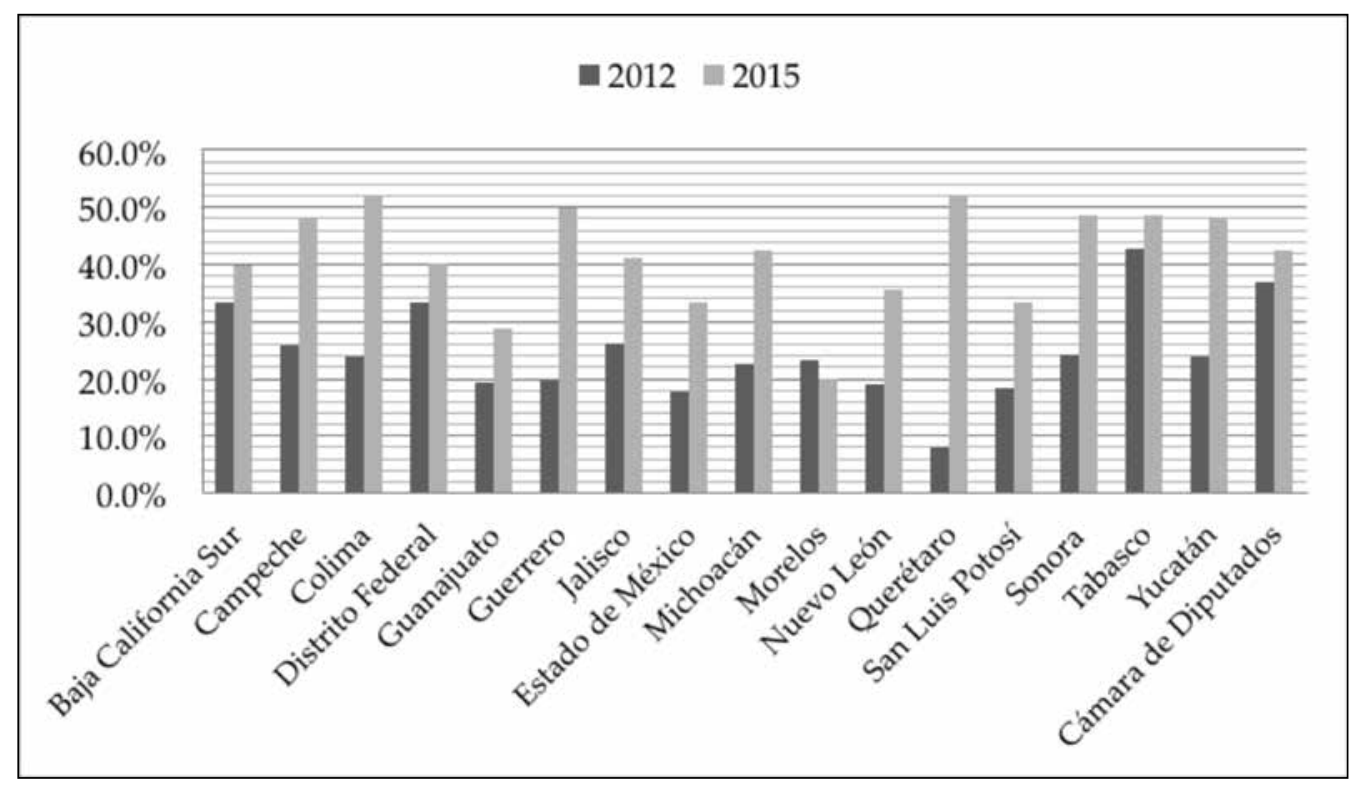

Figura 2. Comparativo de mujeres en los Congresos locales 2012-2015

Fuente: elaborado con base en González, et al., 2016. 


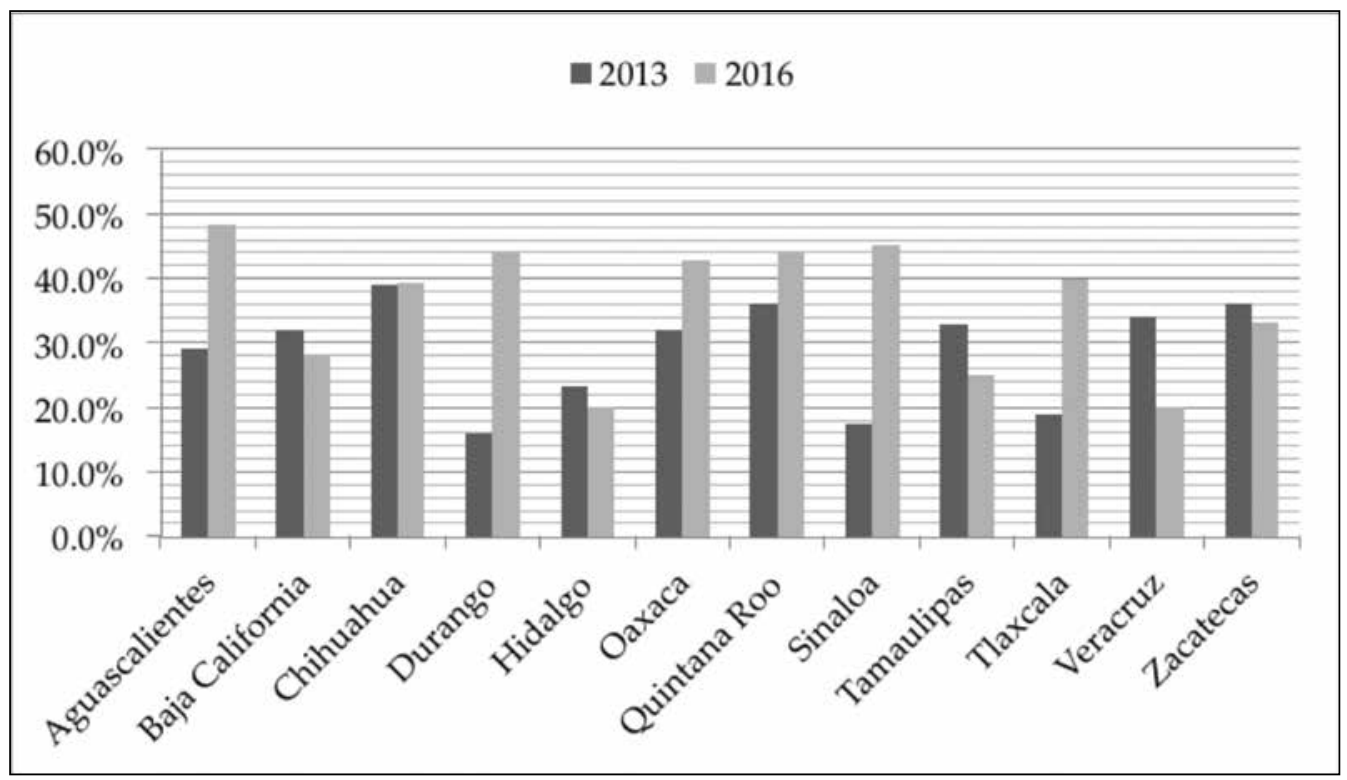

Figura 3. Comparativo de mujeres en los Congresos locales 2013-2016

Fuente: elaborado con base en el Observatorio de la Participación Política de las Mujeres, $2016^{5}$.

Así, con las medidas adoptadas tras la reforma de 2014, un año después, por primera vez la integración del poder legislativo en México se acercó a la paridad de género, en buena medida, gracias a las decisiones del Tribunal Electoral:

El cambio en las exigencias en las normas, sumado al activo papel del Instituto Federal Electoral (hoy Instituto Nacional Electoral) y del Tribunal Electoral del Poder Judicial de la Federación, tanto en la Sala Superior como en las Salas Regionales, han sido claves para ir corrigiendo las prácticas oscuras de muchos partidos

5 El porcentaje de mujeres en los Congresos locales de 2016 podría incrementarse uno o dos escaños con la asignación por representación proporcional, ya que en algunas entidades federativas la declaración de validez de la elección y entrega de documentos oficiales aún se encuentra en proceso. que se han negado de manera sistemática a cumplir con las cuotas o paridad. En este sentido, la práctica más común y más comentada en México ha sido que se ha mejorado la representación de las mujeres "a golpe de sentencias" y de mujeres que exigen cada vez más el respeto de sus derechos políticos (Freidenberg, 2015).

\section{Violencia POlítica de géNERo}

El panorama y los avances descritos en los apartados anteriores contrastan con un fenómeno negativo que se ha incrementado notablemente en México en los últimos años: la violencia política de género, entendida como todas aquellas acciones, conducta u omisiones cometidas contra una mujer o su familia, para restringir, coartar o anular el pleno ejercicio de sus derechos 
político-electorales ( Cerva Cerna, 2014; PAVCM, 2016).

Solamente durante los procesos electorales de 2014-2016, se denunciaron ante las autoridades y los medios de comunicación, agresiones físicas contra la precandidata a una diputación federal en Morelos, Gabriela Pérez Cano, y a su hijo menor de edad (con el mensaje: "Abandona la candidatura"), así como a Yesenia Alamilla Vicente, candidata a la alcaldía del municipio de Reforma, en Chiapas, ambas postuladas por el Partido Acción Nacional; la agresión física y sexual contra la Vocal Ejecutiva electoral Lorena Nava Cervantes, en Juchitán, Oaxaca, y el asesinato de Aidé Nava González, precandidata a la alcaldía de Ahuacuotzingo, Guerrero, postulada por el Partido de la Revolución Democrática. Durante las elecciones en Sonora, en abril de 2015, aparecieron unas mantas en la vía pública con las frases "Las mujeres como las escopetas, cargadas y en el rincón" y "La panocha en las coyotas, jno en palacio!", refiriéndose a las aspiraciones políticas de las mujeres (García Martínez, 2015; PAVCM, 2016). Ya en el marco de las elecciones de 2016, Alberto Silva Ramos, líder del PRI en Veracruz, señaló en una declaración pública que su partido aplicaría "pruebas antiembarazo a las aspirantes a una candidatura" (Sánchez 2015). Además, en 2016 la Fiscalía Especializada para la Atención de Delitos Electorales (Fepade) reportó 38 casos de violencia política de género, denunciados en Baja California, Chiapas, Estado de México, Guerrero, Morelos, Oaxaca, Sonora y Tabasco.

Estos son solo algunos ejemplos que ilustran el panorama de violencia al que se enfrentan las mujeres mexicanas que buscan algún espacio en la política. Los observadores y expertos coinciden: los casos de violencia contra las mujeres involucradas en la política se tornaron más numerosos (Cerva Cerna, 2014, pp. 129-130). Aparentemente, pueden existir tres causas (probablemente, entrelazadas) para ese incremento.

En primer lugar, algunas investigaciones señalan que son una reacción adversa al incremento del número de mujeres en los cargos electivos de distintos niveles. De acuerdo con Daniela Cerva (2014):

La violencia política contra las mujeres se ha exacerbado debido al aumento de su presencia como efecto de la aplicación de la ley de cuotas. Más mujeres en la política se percibe como una amenaza debido a que la tradicional competencia que se daba solo entre varones es suprimida dando paso a que las militantes exijan ser incluidas en los cargos dentro del partido, así como en las candidaturas a elecciones populares. Antes de las cuotas, las mujeres tenían una presencia aislada, sin ningún tipo de poder y en algunos casos -cuando figuraban- era fruto de la concesión que el partido hacía por su relación familiar con la élite o los grupos de poder. Hoy en día, las mujeres comienzan a "estorbar" porque quieren incidir en la política, con programas de trabajo y propuestas independientes (p. 130).

Este fenómeno, también conocido como backlash, es un capítulo mexicano ampliamente descrito por las estudiosas de género: reacciones negativas y frecuentemente violentas contra la participación política de las mujeres (Krook, 2008; Krook y Restrepo, 2016; Baunach, 2002; Superson y Cudd, 2002). 
Una segunda explicación tiene que ver con la ola de violencia generalizada, presente en la región y en México. Lamentablemente, la violencia es hoy parte integral de la vida de las personas en nuestra región $y$, tanto ahora como históricamente, ha sido utilizada como un elemento cotidiano de la lucha política. Ello es especialmente cierto en el caso mexicano, donde la llamada "guerra contra el narcotráfico" iniciada en diciembre de 2006 con el sexenio de Felipe Calderón Hinojosa y que, para agosto de 2015, ha cobrado la vida de más de 164 mil personas, entre ellos 71 alcaldes y cientos de servidores públicos (ABC, 2015; Tonatzin, 2016). En los últimos años, en México se dio un fenómeno de apropiación de los municipios por parte de los grupos del crimen organizado, quienes pretenden controlar esas estructuras para asegurar y facilitar sus operaciones criminales, diversificarlas y que van más allá del tráfico de las drogas, pero también para obtener recursos adicionales de las arcas municipales. Ese escenario implicó mayor presión sobre las autoridades municipales, tanto para los alcaldes como para las agencias policiacas (Valdés Castellanos, 2013; Hope, s. f.).

En ese contexto, es difícil distinguir entre los diversos factores que ubican a las mujeres como víctimas de la violencia. Por ejemplo, el caso de Gisela Mota Ocampo -quien fue asesinada al día siguiente de su toma de posesión como alcaldesa de Temixco, Morelos-, cuya elección y asunción del cargo correspondieron a los cambios en el sistema de seguridad impulsado por el gobernador de Morelos, encaminados a establecer un mando único policial, en un intento de reducir la vulnerabilidad de los policías municipales ante el crimen organizado. Conforme a las denuncias, por lo menos 13 de los 33 alcaldes electos en Morelos recibieron amenazas en los meses previos a su toma de posesión para que no apoyaran el proyecto (Tonatzin, 2016). Ante estos hechos, no queda claro si el asesinato de Gisela Mota tuvo que ver con su género o fue consecuencia de su apoyo abierto al mando único policial y a su firme negativa de ceder ante las exigencias de los grupos criminales. Su caso evidencia lo que señala Piscopo (2016), como una necesidad en los países latinoamericanos para lograr la seguridad y eliminar la violencia política:

[los Estados] deben terminar con la impunidad para quienes perpetran acciones que afectan los derechos a la integridad física de otras personas, independientemente de los motivos con que lleven a cabo dichos actos [...]. Para combatir eficazmente la violencia contra las mujeres en política en América Latina hay que incluir este esfuerzo en un marco más amplio, para seguir con la construcción de instituciones democráticas justas y equitativas (p. 455).

Finalmente, el otro factor explicativo de la ola de violencia política de género puede relacionarse con la nueva perspectiva que las activistas, académicas y los gobiernos están tomando ante ese fenómeno, y que implica ampliar la definición de la violencia política de género. Siguiendo esa línea, Krook y Restrepo (2016) definen la violencia política de género como cualquier acto que impida el pleno ejercicio de los derechos políticos de la mujer. Ante una definición tan amplia, se consideran actos de violencia el incumplimiento con el registro de candidatos respetando la cuota o paridad, el postular a las 
mujeres solamente en los distritos perdedores, no garantizar la equidad en el acceso a los recursos necesarios para realizar campañas electorales, el impedir el ejercicio de un cargo de elección popular por una mujer, comentarios o propaganda sexista, agresiones físicas y otras más. Se puede notar que muchas de esas actividades o comportamientos desde hace tiempo, han sido objeto de pronunciamientos de las autoridades electorales, en especial del TEPJF. Sin haber hablado de violencia política de género, las autoridades electorales jurisdiccionales a lo largo de los años han obligado a los partidos a respetar la normatividad electoral, y a cumplir con la cuota y con otras medidas positivas con el propósito de fortalecer la participación política de las mujeres.

En ese sentido, señala Piscopo (2016): “El hecho de conceptualizar la violencia contra las mujeres en política como crímenes electorales es doblemente problemático. Por un lado, cierra los ojos frente al contexto más amplio de violencia criminal e impunidad rampantes en América Latina”. Y agrega, "y al proceder de esta manera, borra las fronteras entre el sistema de justicia criminal y el de la regulación electoral"' (p. 440), quien sugiere además diferenciar entre el sexismo institucionalizado y las agresiones físicas y psicológicas.

Esas nuevas tendencias conceptuales se ven con claridad en la actividad jurisdiccional del Tribunal Electoral mexicano. Durante 2015 y 2016 el Tribunal en varias sentencias recurrió al empleo del término y de la regulación internacional y local relativa a la violencia política de género. Por ejemplo, en un caso de la magistrada electoral de San Luis Potosí, el TEPJF señaló que el hostigamiento y las dificultades en el desempeño de su cargo que generaban otros magistrados -hombres-, tenían los rasgos de violencia política de género (SUPJDC-4370/2015). En otros casos, analizó la violencia de género en los contenidos de propaganda electoral (SRE-PSC-43/2016 y SUP-REP-88/2016, SUP-REP-90/2016 Y SUP-REP-92/2016, acumulados) y de los materiales de promoción del voto ciudadano, que deben utilizar el lenguaje incluyente (SUP-JDC-1619/2016). También llegó al TEPJF un caso sobre la indebida destitución de una regidora y supuesto acoso laboral. Acerca del primer supuesto, el Tribunal fue competente para resolver, pero en el caso del acoso laboral, informó a las autoridades correspondientes para atender dicha denuncia (ST-JDC-0215/2016).

Como se puede apreciar, la violencia política de género no es nueva, aunque solo recientemente las conductas contrarias a la participación política de las mujeres empezaron a ser analizadas desde la perspectiva de la violencia. Resulta problemático que no haya una definición clara al respecto, porque al no contar con herramientas para su identificación se dificulta que se tomen medidas firmes para evitarla. En el caso mexicano, el Senado de la República aprobó en marzo de 2013 una minuta para tipificarla, pero dicho proyecto de reforma nunca fue ratificado por la Cámara de Diputados, desperdiciando así una oportunidad importante de regular al respecto (Saldaña, 2015; Botello, 2016). Posteriormente se hicieron un par de intentos más para incluirla en las legislaciones mexicanas, pero sin resultados fructíferos. Por ello, para eliminar ese vacío, en marzo de 2016 se publicó el Protocolo para Atender la Violencia Política Contra 
las Mujeres, un instrumento elaborado por diversos organismos como la Secretaría de Gobernación, el Instituto Nacional de las Mujeres, la Comisión Ejecutiva de Atención a Víctimas, el Instituto Nacional Electoral, la Fepade y el TEPJF, entre otros, con el propósito de brindar herramientas para identificación y atención adecuada en los casos de la violencia política de género.

Asimismo, sumándose a esos esfuerzos institucionales, recientemente la Primera Sala de la Suprema Corte de Justicia de la Nación (SCJN) emitió la tesis de Jurisprudencia 22/2016, donde dicta la obligación del juzgador de impartir justicia con perspectiva de género, aun cuando las partes no lo soliciten, para lo cual establece seis elementos que deben observarse: 1) identificar si existe una relación de poder por género entre las partes, 2) evaluar los hechos y las pruebas desechando cualquier estereotipo o prejuicio de género, 3) cuando las pruebas que se aportan no son suficientes para evidenciar la violencia de género, el juez debe ordenar se investigue al respecto, 4) si detecta una situación de desventaja por género, se debe cuestionar la neutralidad del derecho aplicable, así como evaluar el impacto de la posible resolución de acuerdo al contexto de inequidad de género, 5) tomar en cuenta y aplicar los estándares en materia de derechos humanos $y, 6)$ usar siempre lenguaje incluyente, para asegurar la impartición de justicia sin discriminación por motivos de género.

\section{Conclusiones}

Como señala Cerva (2014): “La adopción y aplicación de la cuota de género no implican necesariamente la vigencia de un amplio consenso sobre la existencia de la desigualdad de género" (p. 119). En ese sentido, si bien las cuotas han resultado un mecanismo efectivo para equilibrar la representación política entre las mujeres y hombres en México, no cabe duda que, como mecanismos artificiales que pretenden acelerar cambios sociales, no tienen la capacidad de modificar las conductas sexistas por lo cual no aseguran una plena igualdad entre los géneros. El incremento notable de la violencia política de género en México, es una muestra clara de que el problema de fondo en la inequidad no es numérico, sino que existen importantes resistencias sociales, tanto en la ciudadanía como en la clase política, que encuentran su razón de ser en los persistentes esquemas paternalistas y en la percepción de los hombres de ser propietarios o de tener el derecho único de dominar en la escena política.

Así, el avance de las cuotas se reduce al incremento numérico, pero no logra producir cambios sociales. La apuesta debería hacerse a largo plazo, con políticas públicas integrales que fomenten un cambio en la educación de la sociedad mexicana, a la par de seguir implementando medidas positivas, con el fin de lograr la igualdad en otros ámbitos de la vida social y con ello cambiar posturas. Un buen ejemplo es el caso de los países escandinavos, que han llegado a la paridad por un camino largo, de implementación de políticas públicas y de formación que han generado cambios culturales. Esos cambios, a su vez, llevaron a un paulatino incremento numérico en la representación política de las mujeres. La ruta fast track (o vía rápida) de las cuotas de género tiene la virtud de reducir ese tiempo, pero se debe ser consciente de que no se producen cambios culturales en la sociedad, una necesidad en el caso mexicano. 


\section{REFERENCIAS}

ABC. (2015) La violencia en México provoca más muertos que las guerras de Afganistán e Irak. 11 de agosto. Recuperado de http://www.abc.es/internacional/20150811/abci-guerra-narco-muertos-irak-201508101829.html

Baunach, D. (2002). Progress, opportunity, and backlash: explaining attitudes toward gender-based affirmative action. Sociological Focus, 35(4), 345-362. Recuparado de http://www.jstor.org/ stable/20832179

Botello, B. E. (2016). Legisladoras no logran consenso para sacar reformas de violencia política contra mujeres. La Crónica. México. Recuperado de http://www. cronica.com.mx/notas/2016/952229. html

Cerva Cerna, D. (2014). Participación política y violencia de género en México. Revista Mexicana de Ciencias Políticas y Sociales, 59(222), 117-140. Recuperado de http://www.scielo.org.mx/pdf/ rmcps/v59n222/v59n222a5.pdf

Dahlerup, D. (2006). Women, Quotas and Politics. New York y London: Routledge.

Dahlerup, D., y Freidenvall, L. (2005). Quotas as a 'fast track' to equal representation for women: Why Scandinavia is no longer the model. International Feminist Journal of Politics, 7(1), 26-48.

Dahlerup, D., y Freidenvall, L. (2008). Electoral gender quota systems and their implementation in Europe. Brussels: European Parliament. Recuperado de http:// www.europarl.europa.eu/document/ activities/cont/200903/20090310ATT51390/20090310ATT51390EN.pdf

Freidenberg, F. (2015). ¡Un país (¿ya no?) gobernado por hombres! Los obstáculos que aún dificultan la representación política de las mujeres en México. Revista Digital Democracia y Elecciones. Recuperado de http://democracia-elecciones. mx/abril2015/

García Martínez, A. (2015). Violencia política de género, "punta del iceberg" de la discriminación: especialistas. Revista Proceso. 25 de noviembre. Recuperado de http://www.proceso.com. $\mathrm{mx} / 421656 /$ violencia-politica-de-genero-punta-del-iceberg-de-la-discriminacion-especialistas

González, I. (2015). Fuera 4 partidos en comicios de Chiapas castigados. Excélsior, 19 de julio de 2015. Recuperado de http://www.excelsior.com.mx/ nacional/2015/07/19/1035478\#imagen-2

González Oropeza, M.; Gilas, K. M., y Báez Silva, C. (2016). Hacia una democracia paritaria. La evolución de la participación política de las mujeres en México y sus entidades federativas. México D. F., México: TEPJF

Hope, A. (s. f.). De narcos a mafiosos, del tráfico de drogas al saqueo ciudadano. Narcodata. Animal Político. Recuperado de http://narcodata.animalpolitico. com/de-la-droga-a-la-extorsion/

IDEA. (2002). Mujeres en el Parlamento: más allá de los números. Estocolmo, Suecia: IDEA International.

Krennerich, M. (2009). ¡Mujeres al Parlamento! Sistemas electorales y cuotas de género en la mirilla. En La democracia en su contexto. México D. F., México: UNAM, 2009

Krook, M. L. (2008). La adopción e impacto de las leyes de cuotas de género: una perspectiva global. Mujeres y política. IDEA International, Flacso, Catalonia (pp. 27-60). 
Krook, M. L., y Restrepo Sanín, J. (2016). Género y violencia política en América Latina. Conceptos, debates y soluciones. Política y Gobierno, 23(2), 127-162.

Lau Jaiven, A. (2013). El nuevo movimiento feminista mexicano a fines del milenio. En Rodrigo Laguarda, Jane-Dale Lloyd y Laura Pérez, Género y los procesos de movilización social, México D. F., México: Universidad Iberoamericana

Medina Torres, L. E. (2011). Cuotas electorales de género e integración de Congresos. En Estudios comparados en materia electoral. México D. F., México: UNAMIIJ y TEPJF.

Norris, P. (2006). Recruitment. En R. Katz y W. Crotty (eds.). Handbook of Party Politics. Londres: Sage, pp. 89-108.

PAVCM. (2016). Protocolo para atender la violencia política contra las mujeres. México D. F., México: Segob, TEPJF, INE, Fepade, Inmujeres, CEAV, CONAVIM, FEVIMTRA. Recuperado de http://sitios.te.gob.mx/protocolo_mujeres/media/files/7db6bf44797e749.pdf

Piscopo, J. M. (2016). Capacidad estatal, justicia criminal y derechos políticos. Nueva mirada al debate sobre la violencia contra las mujeres en política. Política y Gobierno, 23(2), 437-458.

Saldaña Pérez, M. L. (2015). Iniciativa con proyecto de decreto por el que se reforman y adicionan diversas disposiciones a la Ley General de Instituciones y Procedimientos Electorales, a la Ley General de Acceso de las Mujeres a una Vida Libre de Violencia y a la Ley General en Materia de Delitos Electorales. Recuperado de http://infosen.senado.gob.mx/ sgsp/gaceta/62/3/2014-11-04-1/assets/ documentos/INIC_PRI_Lucero_VIOLENCIA_POLITICA.pdf

Sánchez, I. (2015). PRI Veracruz aplicará pruebas de embarazo a candidatas. El Financiero. 3 de noviembre. Recuperado de http://www.elfinanciero.com.mx/ nacional/pri-veracruz-aplicara-pruebas-de-embarazo-a-candidatos.html

Santiago Castillo, J. (2011). Justicia electoral. Conflicto político y democratización: 19872009. México D. F., México: TEPJF.

Schnapper, D. (2004). La democracia providencial. Ensayo sobre la igualdad contemporánea. Buenos Aires, Argentina: Homo Sapiens Ediciones.

Superson, A. M., y Cudd, A. E. (eds.). (2002). Theorizing Backlash: Philosophical Reflections on the Resistance to Feminism. Lanham, MD: Rowman \& Littlefield Publishers.

TEPJF. Tribunal Electoral del Poder Judicial de la Federación. (2017). Asuntos resueltos por el Tribunal Electoral del Poder Judicial de la Federación por año. De $1^{\circ}$ de noviembre de 1996 al 13 de julio de 2016. Recuperado de http://www. te.gob.mx/informacion_juridiccional/ estadistica/pdf/G2-Portal\%20SGA.pdf

Tonatzin, P. (2016). Comando ejecuta a Gisela Mota, alcaldesa de Temixco. El Excélsior. 2 de enero. Recuperado de http://www.excelsior.com.mx/ nacional/2016/01/02/1066483

Valdés Castellanos, G. (2013). La historia del narcotráfico en México. México D. F., México: Aguilar.

Woldenberg, J. (2012). Historia mínima de la transición democrática en México. México D. F., México: El Colegio de México. 


\section{ACUERDOS, JURISPRUdENCIAS $Y$ SENTENCIAS}

Acuerdo IEPCO/CG/A-081-2015. Recuperado de http://www.sgg.chiapas.gob. $\mathrm{mx} / \mathrm{po} 2012 /$ archivos/descargas.php? $\mathrm{f}=81-\% 20$ ACUERDO $\% 20$ REGISTRO $\%$ 20CANDIDATOS\%20CUM\%20TEPJF $\% 20030715$.pdf

Acuerdo INE/CG162/2015. Recuperado de http://dof.gob.mx/nota_detalle.php? codigo $=5390614 \&$ fecha $=29 / 04 / 2015$

Acuerdo IMPEPAC/CEE/035/2015. Recuperado de http://impepac.mx/wp-content/ uploads/2014/11/InfOficial/Acuerdos/ IMPEPACCEE0352015.pdf

Sentencia SM-JRC-14/2014. Recuperado de http://portal.te.gob.mx/colecciones/ sentencias/html/SM/2014/JRC/SMJRC-00014-2014.htm

Sentencia ST-JRC-21/2015. Recuperado de http://portal.te.gob.mx/colecciones/ sentencias/html/ST/2015/JRC/ST-JRC00021-2015.htm

Sentencia ST-JRC-215/2016. Recuperado de http://www.te.gob.mx/EE/ST/2016/JDC /215/ST_2016_JDC_215-573791.pdf

Sentencia SUP-JDC-2580/2007 y sus acumulados SUP-JDC-1/2008 y SUP-JDC-12/ 2008. Recuperado de http://portal.te.gob. $\mathrm{mx} /$ colecciones/sentencias/html/SUP /2007/JDC/SUP-JDC-02580-2007.htm

Sentencia ST-JDC-278/2015, ST-JDC-279/ 2015 y ST-JDC-280/2015. Recuperado de http://portal.te.gob.mx/colecciones/ sentencias/html/ST/2015/JDC/ST-JDC -00241-2015.htm

Sentencia SUP-JDC-12624/2011. Recuperado de http://portal.te.gob.mx/coleccio nes/sentencias/html/SUP/2011/JDC/ SUP-JDC-12624-2011.htm
Sentencia SUP-JDC-1619/2016. Recuperado de http://portal.te.gob.mx/colecciones/ sentencias/html/SUP/2016/JDC/SUPJDC-01619-2016.htm

Sentencia SUP-JDC-4370/2015. Recuperado de http://portal.te.gob.mx/colecciones/ sentencias/html/SUP/2015/JDC/SUP_ JDC-04370-2015.htm

Sentencia SUP-JRC-680/2015. Recuperado de http://portal.te.gob.mx/colecciones/ sentencias/html/SUP/2015/JRC/SUPJRC-00680-2015.htm

Sentencia SRE-PSC-43/2016. Recuperado de http://portal.te.gob.mx/colecciones/ sentencias/html/SRE/2016/PSC/SREPSC-00043-2016.htm

Sentencia SUP-RAP-134/2015. Recuperado de http://portal.te.gob.mx/colecciones/ sentencias/html/SUP/2015/RAP/SUPRAP-00134-2015.htm

Sentencia SUP-REC-294/2015. Recuperado de http://portal.te.gob.mx/colecciones/ sentencias/html/SUP/2015/REC/SUPREC-00294-2015.htm

Sentencia SUP-REC-936/2014. Recuperado de http://portal.te.gob.mx/colecciones/ sentencias/html/SUP/2014/REC/SUPREC-00936-2014.htm

Sentencia SUP-REC-756/2015 y SUP-REC762/2015, acumulados. Recuperado de http://www.te.gob.mx/Infor macion_juridiccional/sesion_publica/eje cutoria/ sentencias/SUP-REC-07 56-2015.pdf

Sentencia SUP-REC-694/2015. Recuperado de http://portales.te.gob.mx/genero/sites /default/files/SUP-REC-0694-2015.pdf

Sentencia SUP-REP-88/2016, SUP-REP-90 /2016 Y SUP-REP-92/2016, acumulados. Recuperado de http://portal.te.gob.mx/ colecciones/sentencias/html/SUP/2016/ REP/SUP-REP-00088-2016.htm 
Tesis de Jurisprudencia 22/2016. Recuperado de http://200.38.163.178/sjfsist/ Paginas/DetalleGeneralV2.aspx?Epo $\mathrm{ca}=1 \mathrm{e} 3 \mathrm{e} 10000000000 \&$ Apendice $=100$ 0000000000\&Expresion $=$ juzgar $\% 2520$ con $\% 2520$ perspectiva $\% 2520 \mathrm{de} \%$ $2520 \mathrm{~g} \% 25 \mathrm{C} 3 \% 25 \mathrm{~A} 9$ nero\&Dominio= Rubro,Texto\&TA_TJ $=2 \&$ Orden $=1 \&$ Clase $=$ DetalleTesisBL $\&$ Num TE $=6$ $\&$ Epp $=20 \&$ Desde $=-100 \&$ Hasta $=-100 \& I n$ dex $=0 \&$ InstanciasSeleccionadas $=6,1,2$, 50,7\&ID=2011430\&Hit=1\&IDs=201143
0,2009998,2010005,2008545,2005793,2 $005794 \&$ tipoTesis $=\&$ Semanario $=1 \&$ ta$\mathrm{bla}=\&$ Referencia $=\&$ Tema $=$

\section{LEGISLACIONES}

COFIPE. Código Federal de Instituciones y Procedimientos Electorales.

CPEUM. Constitución Política de los Estados Unidos Mexicanos.

LEGIPE. Ley General de Instituciones y Procedimientos Electorales.

LGPP. Ley General de Partidos Políticos. 